\title{
Generation, control and erasure of dual LIPSS in germanium with fs and ns laser pulses
}

\author{
Noemi Casquero ${ }^{\dagger_{*}^{*}}$, Yasser Fuentes-Edfuf ${ }^{\dagger}$, Raul Zazo ${ }^{\dagger}$, Javier Solis ${ }^{\dagger}$ and Jan Siegel ${ }^{\dagger^{*}}$ \\ 'Laser Processing Group, Instituto de Óptica, IO-CSIC, Serrano 121, 28006 Madrid \\ KEYWORDS: LIPSS, Femtosecond laser, Nanosecond laser, Ripples, HSFL, LSFL
}

\begin{abstract}
Laser-induced periodic surface structures (LIPSS) can readily be fabricated in virtually all types of materials and benefit from an efficient parallel patterning strategy that exploits self-organization. The wide range of different LIPSS types with different spatial scales and symmetries is continuously growing, addressing numerous of applications. Here, we report on the formation of two fundamentally different types of LIPSS on germanium upon exposure to femtosecond laser pulses $(\lambda=800 \mathrm{~nm}, 130 \mathrm{fs})$, featuring different periods and orthogonal orientations. On the one hand, the well-known low-spatial frequency LIPSS (LSFL) with a period $\approx \lambda$ and perpendicular orientation to the laser polarization are formed, which can be extended homogeneously in 2D by sample scanning. Additionally, extremely smooth ripples with a period $\approx \lambda / 2$ and parallel orientation were generated at lower pulse numbers. We show that this new kind of ripples, named HSFL- $\|$, can be superimposed onto LSFL by increasing the pulse number, forming complex dual LIPSS with nanohill-like morphology. While exposure to multiple nanosecond laser pulses is found to trigger also the formation of LSFL, HSFL- $\|$ cannot be formed under these conditions, which points out the role of ultrafast excitation in the formation of the latter. By performing time-resolved reflectivity measurements, we are able to resolve the melting and solidification dynamics, revealing melting of a very shallow surface layer $(<20 \mathrm{~nm})$ and melt durations of a few ns for both pulse durations pulses at the fluences employed for LIPSS formation. Finally, we demonstrate erasure of both types of LIPSS by exposure to single nanosecond pulses at high fluences, which paves the way for erasable multi-level data storage.
\end{abstract}

\section{INTRODUCTION}

Laser-induced periodic surface structures (LIPSS) have gained considerable attention from researchers and industry due to their surface functionalization potential [1]. They can be produced by a single-step method, which enables efficient and flexible fabrication for numerous applications. LIPSS can readily be formed in metals [2], semiconductors [3] and dielectrics [4] by exposure to multiple short or ultrashort laser pulses. Their morphology strongly depends on many parameters [5-8] related both to the irradiation conditions [9,10] (laser fluence, wavelength, polarization, repetition rate, environment, and processing speed) and material properties. LIPSS are typically distinguished by their spatial period. Low-spatial frequency LIPSS (LSFL), with a period close to laser wavelength, are fairly well understood in most materials $[11,12]$. Their origin is generally interpreted as the result of the interaction between the incident light and an electromagnetic surface wave; the latter being either scattered light or surface plasmon polaritons 
(SPP) propagating at the air/material interface. This interaction causes a periodic modulation of the spatial intensity distribution, in which processes like melting, ablation, or even oxidation [13] are triggered preferentially at the local intensity maxima, imprinting a permanent ripple pattern onto the sample surface after multiple laser pulses irradiation.

In contrast, for high-spatial frequency LIPSS (HSFL), having periods much shorter than the laser wavelength, large discrepancies are often observed between existing models and the experimentally measured features like period and orientation. The possible formation mechanisms of these ripples include interference along with transient changes in the optical properties during laser irradiation [14], surface second harmonic generation [15], excitation of surface plasmon polaritons [11] and self-organization [16].

The fabrication of LIPSS in semiconductors is of particular interest due to their dominant role in the electronics and photovoltaic industries, in which flexible and rapid nanostructuring strategies are desirable. It has been shown that features as small as $12 \mathrm{~nm}(\lambda / 66)$ on silicon surfaces can be fabricated via a LIPSS-based process, employing irradiation with orthogonally polarized double femtosecond laser beams [17] While most of the published work has been concentrated on silicon $[5,14,17-27]$, several works have been focused on germanium (Ge) $[15,22,28-36]$. One of the early works reporting LIPSS generation on Ge was published by Young et al., who employed ns pulses [22] to generate LSFL at different angles of incidence, reporting a strong angle dependence of their period and orientation. Virtually all of the later works on LIPSS in Ge are performed with ultrashort laser pulses. This strong change in the excitation conditions, generating higher electron densities, faster melting and thus steeper initial temperature gradients, might be underlying reason for the different type of ripples observed in those works. Le Harzic et al. [15], for instance, reported HSFL ripples in Ge oriented perpendicular to the laser polarization, with an average period of 160 $\mathrm{nm}$ upon irradiation with femtosecond laser pulses at $750 \mathrm{~nm}$ wavelength. Similarly, using fs laser pulses at $800 \mathrm{~nm}$, Lin et al. [33] reported HSFL in Ge with a period close to $95 \mathrm{~nm}$, also oriented perpendicular to the polarization and superimposed on LSFL with period of $500 \mathrm{~nm}$, also perpendicular to the polarization. In contrast, Austin et al. [34] reported HSFL in Ge aligned parallel to the polarization with a period of $850 \mathrm{~nm}$ using mid-IR laser irradiation at $2400 \mathrm{~nm}$ and normal incidence. Borowiec et al. [23] observed LSFL in Ge only at a very long laser wavelength $(2100 \mathrm{~nm})$ and reported no evidence of HSFL or LSFL at $800 \mathrm{~nm}$ or intermediate wavelengths. However, the same authors reported LSFL and HSFL in GaP at $800 \mathrm{~nm}$, with respective periods of $680 \mathrm{~nm}$ and $170 \mathrm{~nm}$ and perpendicular orientation to the laser polarization. The broad spectrum of different results illustrates the still limited understanding of the mechanisms and main parameters that control the triggering HSFL formation in Ge.

In this work, we report on the formation of two fundamentally different types of LIPSS in Ge by exposure to fs laser irradiation, featuring different periods and orthogonal orientations.

Additionally, we have investigated the differences in the LIPSS morphologies induced by femtosecond or nanosecond laser pulses. Lastly, we demonstrate a simple method for LIPSS erasure by exposure to single nanosecond pulses.

\section{MATERIALS AND METHODS}

The material for studying ripple formation was commercial polished crystalline germanium $<100>$ wafers (Ga-doped, $160 \mu \mathrm{m}$ thick). For sample irradiation, a Ti:Sapphire laser system consisting of 
a mode-locked oscillator and a regenerative amplifier was used. The system delivered pulses of $130 \mathrm{fs}$ duration at a central wavelength of $800 \mathrm{~nm}$, at $100 \mathrm{~Hz}$ repetition rate. This system could also provide pulses in the ns regime with a temporal duration of $8 \mathrm{~ns}$. The pulse energy was externally adjusted by means of a variable attenuator formed by a half-waveplate and a polarizing beam splitter. A mechanical shutter was used to control the number of laser pulses incident on the sample while a half-waveplate allowed to select the orientation of the linearly polarized laser light. The beam was sent through a circular aperture of $3.5 \mathrm{~mm}$ in diameter, before being focused on the sample surface by a lens with $150 \mathrm{~mm}$ focal length. A three-dimensional translation stage was used to move the sample and a rotating platform to select the incidence angle of the beam. Experiments were carried out at $0^{\circ}$ and at $52^{\circ}$. The laser spot diameter was determined experimentally, following the method used by Liu [37], to be Gaussian with radial symmetry at normal incidence, having a $1 / \mathrm{e}^{2}$ diameter of $\mathrm{d}=58.96 \mu \mathrm{m}$. The fluence values quoted in the paper correspond to the peak fluence $F$, calculated as $F=2 E / A$, with $E$ being the pulse energy and $A=\pi d^{2} / 4$. All experiments were performed in air.

For analyzing the temporal evolution of the surface reflectivity upon laser excitation, a real-time reflectivity setup, with a temporal resolution of $2 \mathrm{~ns}$ and a measured rise-time of a reflectivity change of $\approx 600 \mathrm{ps}$, was used [26]. The setup is composed of a continuous wave probe laser at a wavelength of $532 \mathrm{~nm}$ focused by a microscope objective at normal incidence onto the center of the pump laser-excited region, the spot size of pump laser is $\approx 1 \mu \mathrm{m}$, coinciding with the peak pump fluence. The reflected probe beam is sent to an avalanche photodiode, protected by a short pass filter from pump light scatter. The signal of the photodiode is displayed by an oscilloscope and recorded.

The surface morphology was analyzed by means of an optical microscope (OM) supplied with monochromatic light illumination at $460 \mathrm{~nm}$ and a field emission scanning electron microscope (SEM). The period of the LIPSS was measured by performing a 2D-FFT (fast Fourier transform) of the recorded OM and SEM images, employing a custom Matlab code. The surface topography was measured with an atomic force microscope (AFM) in tapping mode.

\section{RESULTS AND DISCUSSION}

\section{$\underline{\text { LIPSS formation upon fs laser pulse irradiation }}$}

The Ge surface was irradiated at normal incidence with different combinations of laser fluence and number of fs pulses, thus obtaining individual processed spots. Figure 1 shows the OM images of the different resulting spots for selected combinations of parameters. The fluence $\mathrm{F}$ was varied from $106-139 \mathrm{~mJ} / \mathrm{cm}^{2}$ and the pulse number $\mathrm{N}$ from 50 to 300 . For $\mathrm{F}=106 \mathrm{~mJ} / \mathrm{cm}^{2}$ and $\mathrm{N}=75$, the irradiated spot features fine ripples oriented parallel to the polarization of the incident laser. Increasing the pulse number, these ripples become more pronounced for $N=100$ and $N=200$. At $\mathrm{N}=300$, it can be seen that the ripple orientation has changed, being now perpendicular to the laser polarization and displaying a strong optical contrast and a coarser spacing compared to lower pulse numbers. For a slightly higher fluence $F=128 \mathrm{~mJ} / \mathrm{cm}^{2}$ and $\mathrm{N}=75$, fine ripples appear just in an external ring of the irradiated spot whereas the central disk appears featureless. This behavior shows that ripple formation is limited to a small fluence window, namely $\Delta \mathrm{F}=22 \mathrm{~mJ} / \mathrm{cm}^{2}$, similar to the case of amorphous-crystalline LSFL observed in silicon under similar processing conditions [24]. 


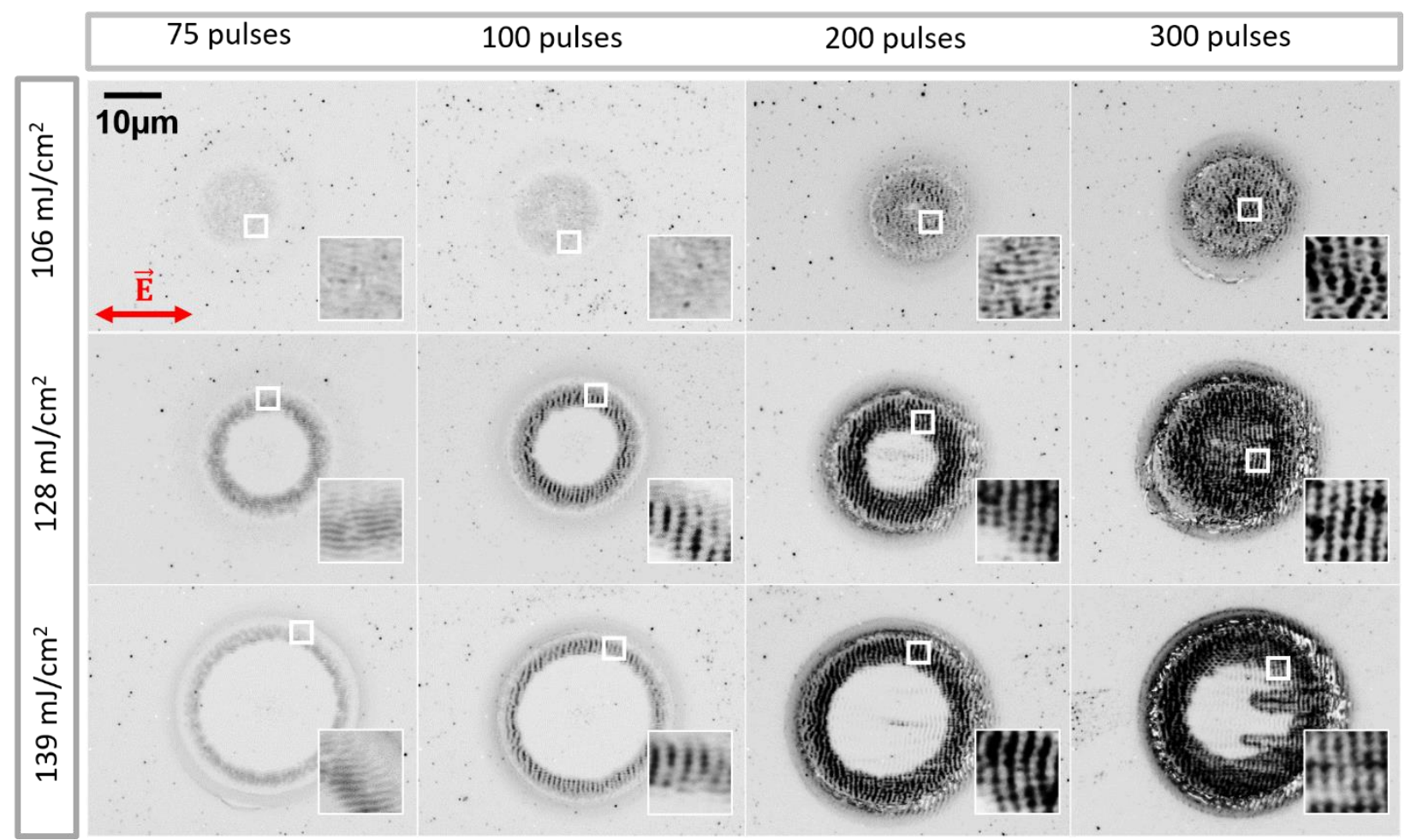

Figure 1. Optical micrographs of fs laser irradiated regions at different laser fluences $F$ (rows) and number of pulses $\mathrm{N}$ (columns). The red arrow indicates the laser polarization direction. Each frame contains a magnification of a representative region that contains ripples. Note the appearance of a new kind of LIPSS oriented parallel to the polarization (HSFL- $\|$ ).

For $\mathrm{N}=100$, this external ring shows two kinds of ripples, fine horizontal ripples and coarser ripples oriented perpendicular to laser polarization, while the central disk remains featureless. Further increasing the number of pulses to $\mathrm{N}=200$, the horizontal ripples disappear while the vertical ones remain. Interestingly, in this case the central disc shows weak signs of vertical ripples extended from the outer ring, but with reduced contrast. For $\mathrm{N}=300$, the entire irradiated spot is covered with vertical ripples. For the highest laser fluence studied, $F=139 \mathrm{~mJ} / \mathrm{cm}^{2}$, the ripple evolution follows the same behavior: for low pulse numbers, the external ring contains horizontal ripples, which evolve upon pulse number increase into vertical or dual type ripples covering most of the spot.

In order to obtain high-resolution images of the morphology of the generated ripples and study their evolution with pulse number, we have performed SEM on selected spots. Figure 2a shows the image of a spot generated at $\mathrm{F}=128 \mathrm{~mJ} / \mathrm{cm}^{2}$ and $\mathrm{N}=100$, featuring the characteristic outer ring that contains ripples oriented parallel to the laser polarization. The magnification of a ring region shown next to it (Figure $2 b$ ) reveals that the ripples are extraordinarily smooth, which suggests that the self-organization process was dominated by melting, material displacement and re-solidification, involving no (or only minimal) material removal. This conclusion is supported by the very sharp transition in the horizontal direction from a non-modified region to the ripple region, without signs of an ablation rim, as it would be expected for ablative LIPSS. The ripple 
period was determined to be $\Lambda_{\|}=400 \pm 50 \mathrm{~nm}$, half of the laser wavelength $(\Lambda=800 \mathrm{~nm})$. Their maximum modulation depth is $\mathrm{d}<30 \mathrm{~nm}$, as it can be seen in Figure 2c, displaying an AFM map of a small region of the outer ring.

The above features have not been reported before for any type of ripples in semiconductors. LSFL typically reported in Ge and Si are always oriented perpendicular to the laser polarization and have a period close to the laser wavelength $[5,33]$. The observed behaviour is also fundamentally different from the recently reported amorphous-crystalline LSFL-type in silicon, featuring perpendicular orientation and exact-wavelength periodicity [24]. In this context, it is worth noting that we have performed micro-Raman measurements (excitation at $532 \mathrm{~nm}$, spatial resolution $1 \mu \mathrm{m}$ ) on the irradiated regions in order to investigate if the amorphous phase of Ge might be present. Neither in the annular ring structure containing HSFL- $\|$ and LSFL nor in the central disk we could detect Raman bands, except the one corresponding to the crystalline phase (results not shown). Moreover, no Raman bands corresponding to $\mathrm{GeOx}$ were observed, confirming that no surface oxidation was induced by the laser irradiation even at high pulse numbers. The latter result is relevant for classifying the ripple type, since several works have reported the existence of so-called LSFL- $\|$, attributed to so-called by radiation remnants. This is the case for instance in Ref. [22], who reported LSF- $\|$ in Ge upon ns-laser irradiation but only at elevated angles of incidence and resulting in periods larger than the laser wavelength. Another work reported LSFL- $\|$ imprinted at the interface of steel coated with a $100 \mathrm{~nm}$ thick oxide layer, attributing it to the scattering of the laser radiation at the nanoscale surface roughness and the propagation through a superficial oxide layer [38]. The orientation of such structures is parallel to the polarization and the period scales as $\Lambda \approx \lambda / \mathrm{n}$, with $\mathrm{n}$ being the refractive index of the irradiated material. However, the absence of Raman bands corresponding to $\mathrm{GeOx}$ in our case supports our classification of the ripple structures as HSFL-\|.

The characteristics of the ripples reported here also differ strongly from those of HSFL reported in Ge and $\mathrm{Si}$, which typically show much shorter periods $\left(\Lambda_{\mathrm{HSFL}} \approx 100 \mathrm{~nm}\right.$ for Ge and Si [33]), perpendicular orientation with respect to the laser polarization (same as LSFL) [33,39], and appear at higher pulse numbers than LSFL [33] rather than at lower ones. These fundamental differences to existing ripple structures reported, together with their particularly smooth morphology with almost no ablation, suggests the existence of a new kind of LIPSS, named hereafter HSFL- $\|$, obeying a different formation mechanism, on which we will shed more light in the following sections.

The influence of pulse number is illustrated in Figure 2d, displaying the SEM image of a spot generated at $\mathrm{F}=128 \mathrm{~mJ} / \mathrm{cm}^{2}$ and $\mathrm{N}=200$. Here, well-pronounced vertical ripples, with a larger period are observed. Both, period $\Lambda_{\perp}=790 \pm 30 \mathrm{~nm}$ and perpendicular orientation to the laser polarization are consistent with the well-known LSFL reported in semiconductors [5,33]. Interestingly, upon magnification (Figure 2e) it can be seen that the structures formed are rather complex and still contain the signature of the HSFL-\| structures, forming dual LIPSS. In particular, a splitting of each individual vertical ripple is observed, effectively forming an array of nanohills, with a vertical spacing equal to $\Lambda_{\|}=400 \pm 50 \mathrm{~nm}$ and a horizontal spacing of $\Lambda_{\perp}=803 \pm 20 \mathrm{~nm}$. As it can be seen in Figure $2 \mathrm{f}$, the maximum modulation depth of these dual LIPSS structures is considerably larger than for HSFL-\| structures, namely $\mathrm{d}<90 \mathrm{~nm}$, which is qualitatively consistent 
with the high pulse number used that cause an increase in surface roughness and modulation depth[40].
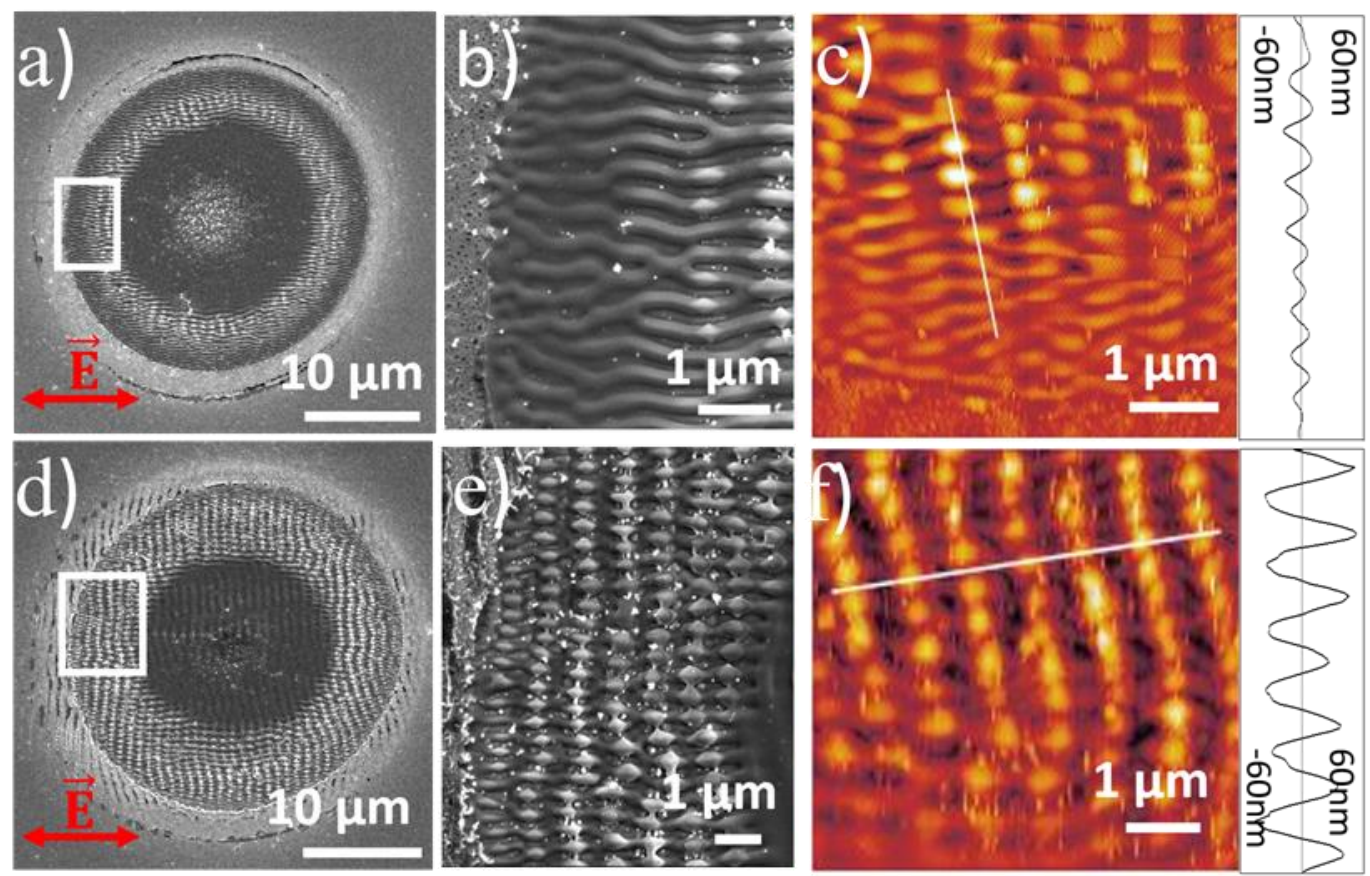

Figure 2. Transition from HSFL-\| to dual LIPSS: SEM and AFM images of fs laser irradiated regions at $\mathrm{F}=128 \mathrm{~mJ} / \mathrm{cm}^{2}$ for different pulse numbers $\mathrm{N}=100$ (a)-c)) and $\mathrm{N}=200$ (d)-f)). The red arrow indicates the laser polarization direction valid for all images. b),d) are magnifications of the white squares marked in the SEM images a),d). The white lines in the AFM images c),f) indicate the positions of the topography profiles plotted next to the images.

A powerful method to improve fringe homogeneity and alignment, as well as to extend fringes in $2 \mathrm{D}$, is to scan the laser spot over the surface at a well-defined repetition rate and speed [24,25]. Figure 3 shows regions of a surface irradiated while the sample was scanned in the horizontal direction under the incident fs laser beam operating at $100 \mathrm{~Hz}$ repetition rate, giving rise to laterally extended LSFL. Figure 3a shows highly homogeneous LSFL formed upon irradiation with horizontal polarization, yielding a period $\Lambda_{\perp}=800 \pm 30 \mathrm{~nm}$. Upon rotation of the beam polarization, the ripple orientation rotates as well and the period remains unchanged (Figure $3 b$ ), although the ripple homogeneity clearly degrades. This fact demonstrates that sample scanning parallel to the polarization vector enables the formation of homogeneous and periodic grating structures.

As already reported for amorphous crystalline ripples formed in Si [25], this scanning strategy incubates a fringe structure along the scan direction before the actual laser spot passes, and thus triggers melting of periodic ripples, which greatly facilitates the formation of homogeneous, large scale periodic structures. 


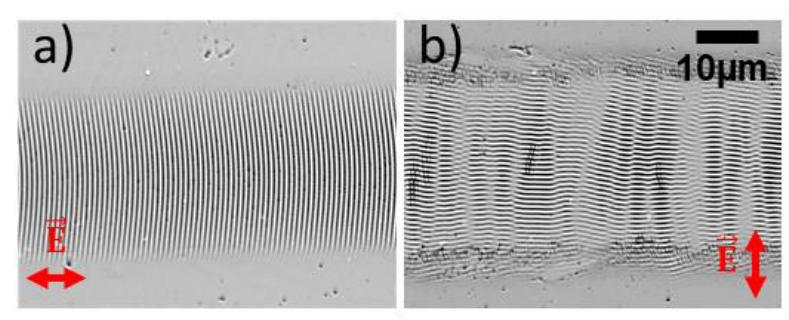

Figure 3. Optical micrographs of 2D extended ripples by sample scaning upon fs laser irradiation at $100 \mathrm{~Hz}$. a) scan speed $=0.050 \mathrm{~mm} \mathrm{~s}^{-1}$ (transversal LIPSS); b) scan speed $=0.035 \mathrm{~mm} \mathrm{~s}^{-1}$ (lengthwise LIPSS). The polarization orientation is indicated by the red arrow.

\section{LIPSS generation upon ns laser pulse irradiations}

In order to investigate the influence of the pulse duration on LIPSS formation, a similar study using different laser fluences and pulse numbers was carried out using operating the laser system at $8 \mathrm{~ns}$ pulse duration, still at $800 \mathrm{~nm}$ wavelength and normal incidence. In this case, the fluence range explored was Fns $=\left[149 \mathrm{~mJ} / \mathrm{cm}^{2}-298 \mathrm{~mJ} / \mathrm{cm}^{2}\right]$ and the pulse number range was $\mathrm{N}_{\mathrm{ns}}=[100$ $-300]$, the results being shown in Figure 4. Globally, it can be stated that for nanosecond pulse irradiation, LSFL aligned perpendicular to the polarization direction are mainly observed. Moreover, as the fluence is increased, the LSFL appear in an outer ring surrounding the of the spot in a similar arrangement as in the results obtained with fs laser pulses. Yet, in this case, the central disk is less homogenous compared to fs pulses, and shows clear signs of thermal effects, ablation and corrugation due to the longer pulse duration.

The fact that the lowest fluence $\mathrm{F}_{\mathrm{ns}}=149 \mathrm{~mJ} / \mathrm{cm}^{2}$ required to produce rippled structures is only $40 \%$ higher than the one used for fs pulses $\left(\mathrm{F}_{\mathrm{fs}}=106 \mathrm{~mJ} / \mathrm{cm}^{2}\right)$ illustrates the importance of linear absorption of the laser light by the material, even for excitation with ultrashort laser pulses. The underlying reason is the relatively short penetration depth of the laser light in crystalline Ge $\left(\mathrm{L}_{\text {opt }}(800 \mathrm{~nm})=198 \mathrm{~nm}\right)$. Despite the similar fluence and pulse number range for $\mathrm{ns}$ and fs laser pulses to form periodic LSFL, considerable differences were though observed in other aspects. Most notably, the absence of HSFL-\| structures for ns pulses, even when extending the parameter range (pulse number and fluence). This suggests the importance of ultrafast excitation and/or related non-linear effects in the formation of HSFL-\|. Whether an indirect effect of ultrashort laser pulse excitation, the generation of a very steep temperature gradient and resulting faster melting and solidification dynamics, might also contribute to the formation of HSFL-\| will be investigated in the next section. 


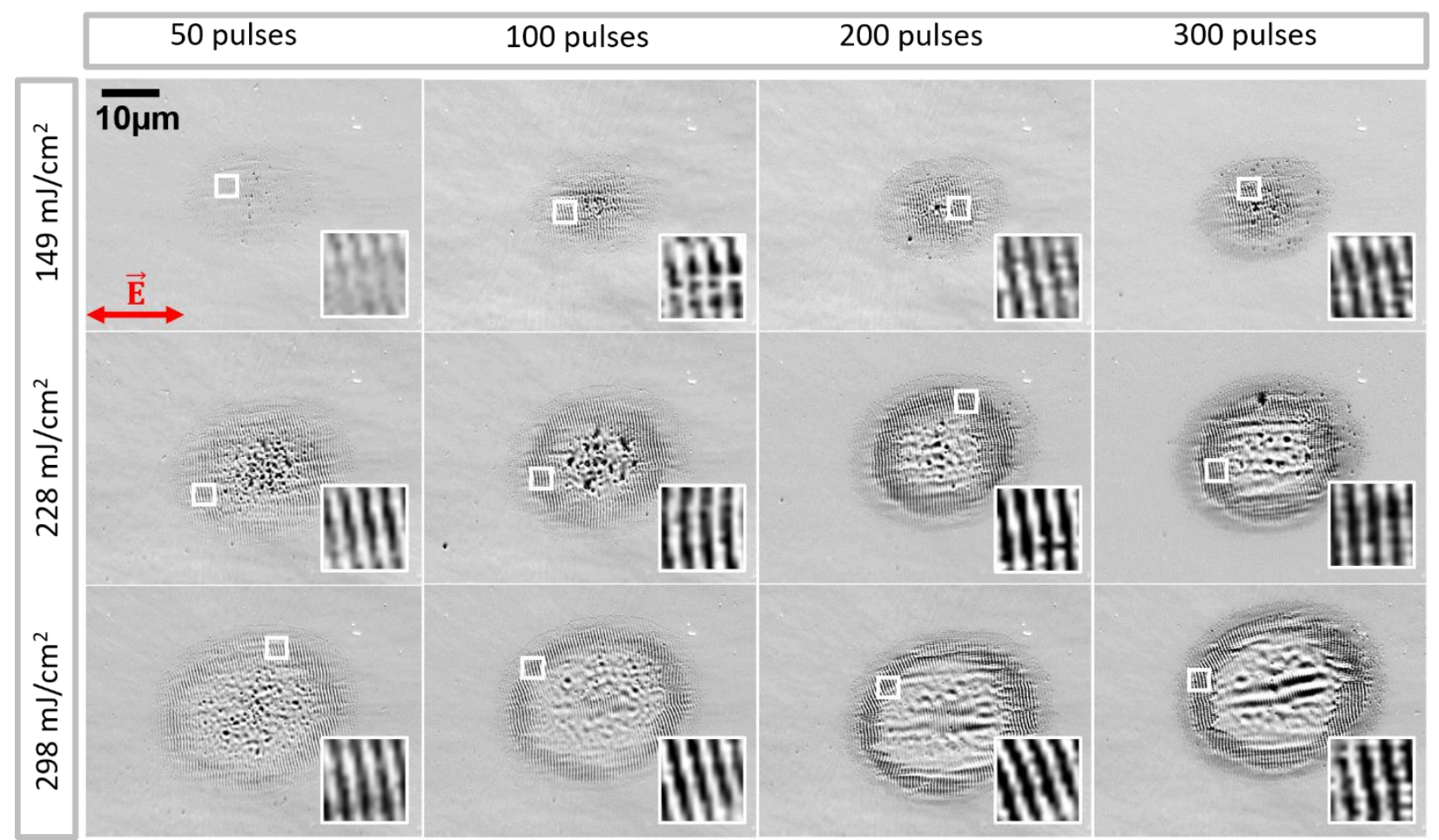

Figure 4. Optical micrographs of ns laser irradiated regions at different laser fluences $\mathrm{F}$ (rows) and number of pulses $\mathrm{N}$ (columns). The red arrow indicates the laser polarization direction. Each frame contains a magnification of a representative region that contains ripples. Compared to the results obtained with fs pulses, the most notable differences are the absence of horizontal HSFL- $\|$ and the appearance of thermal effects, ablation and corrugation in the central disk.

\section{Time-resolved reflectivity measurements}

We have investigated the temporal evolution of laser-induced heating, melting and solidification processes at the germanium surface upon fs and ns laser irradiation, employing time-resolved reflectivity measurements. Of particular interest to us was to determine the presence and lifetime of the molten phase in the fluence regime used to generate LIPSS structures. It has to be noted, though, that the fluence values used in this experiment cannot be compared directly to the results shown in Figures 2-4, since the irradiation angle is different $\left(\theta=52^{\circ}\right.$ for time-resolved experiments, $\theta=0^{\circ}$ for Figures 2-4), and thus the absorbed energy of the p-polarized pulses. In order to identify the correct laser fluence for the time-resolved experiments, we have first performed a systematic study of static irradiations at $\mathrm{N}=200$ and $\theta=52^{\circ}$, varying the laser fluence. By inspection of the fabricated spots by optical microscopy, we identified the spots that most that closely resembled those shown in Figure 2 for fs pulses $\left(F\left(0^{\circ}, f s\right)=106 \mathrm{~mJ} / \mathrm{cm}^{2}\right)$ and Figure 4 for ns pulses $\left(\mathrm{F}\left(0^{\circ}, \mathrm{ns}\right)=149 \mathrm{~mJ} / \mathrm{cm}^{2}\right)$. While considerable differences in the LIPSS structures were observed for the two different irradiation angles (as expected from the Sipe theory and reported in literature [22]), this method allows an identification of the optimum laser fluence for LIPSS 
formation at $52^{\circ}$. The values obtained were $\mathrm{F}_{\mathrm{fs}}\left(52^{\circ}\right)=74 \mathrm{~mJ} / \mathrm{cm}^{2}$ and $\mathrm{F}_{\mathrm{ns}}\left(52^{\circ}\right)=137 \mathrm{~mJ} / \mathrm{cm}^{2}$, which were used for the time-resolved experiments shown in the following.

Figure 5 shows the evolution of the surface reflectivity (normalized to the sample reflectivity before irradiation) upon irradiation with single pulses at the two different pulse durations. For fs laser excitation, a rapid and strong reflectivity increase, limited by the rise-time of the detection system (600 ps), is observed, followed by slightly slower decrease, reaching quickly a reflectivity value similar to the initial value. This behavior is consistent with melting and solidification of a thin surface layer, since the reflectivity of the molten phase of $\mathrm{Ge}$ is much higher than that of the solid phase [35]. It can be seen that the measured reflectivity maximum $\Delta \mathrm{R}_{\max }(\mathrm{fs})=1.37$ is smaller than the calculated reflectivity increase for deep melting of Ge $\left(\Delta R_{\text {molten }}=1.46\right)$. We have ensured that the expected reflectivity level for complete melting can be achieved when increasing the fluence only slightly, to $\mathrm{F}_{\mathrm{fs}}\left(52^{\circ}\right)=78 \mathrm{~mJ} / \mathrm{cm}^{2}$ (data not shown). This comparison demonstrates than only a thin layer is molten at this laser fluence, with a lower thickness value than an optically thick layer at the probe light wavelength $\left(\mathrm{d}_{\text {ОтL }}(532 \mathrm{~nm}) \approx 20 \mathrm{~nm}\right)$ [28]. This result is important for the understanding of the LIPSS formation mechanism, clarifying that LIPSS formed upon irradiation with fs laser pulses emerge from a very shallow pool of molten Ge. This result is consistent with time-resolved measurements in Ge upon ns laser irradiation reported by Ehrlich et al [30], who found that efficient ripple growth occurs when the melt depth is less than $20 \mathrm{~nm}$.

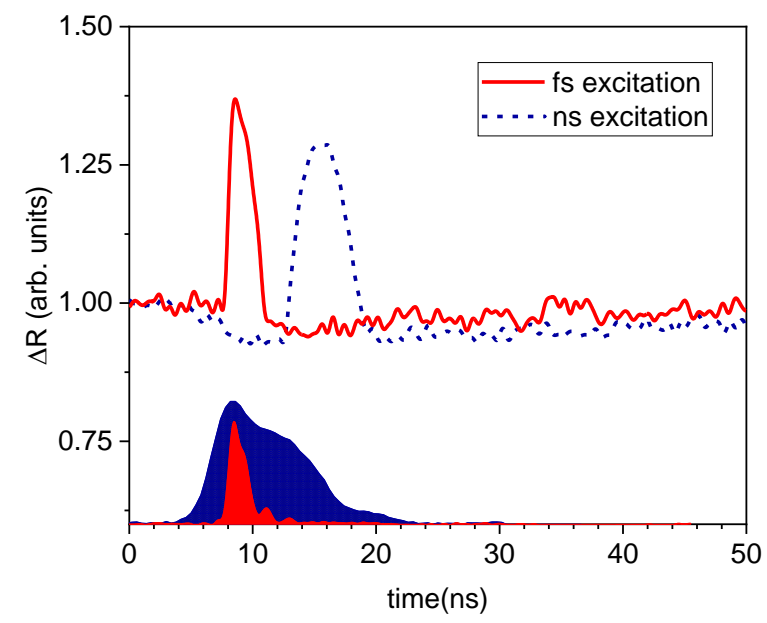

Figure 5. Measured evolution of the normalized surface reflectivity change $\Delta \mathrm{R}$ with time upon exposure to single fs and ns laser pulses at the laser fluences that lead to the generation of LIPSS in each case (see text for details). Filled red and blue areas correspond to the measured temporal profile and position of the fs and ns excitation pulses, respectively.

In the case of the ns laser excitation, the reflectivity response also displays a fast increase and decrease, consistent with the melting of a surface layer that is thinner than $\mathrm{d}_{\mathrm{OTL}}(532 \mathrm{~nm}) \approx 20 \mathrm{~nm}$. However, in this case the melting process is delayed with respect to the onset of the excitation pulse (included in Fig. 5 as a blue shaded curve at the bottom), starting at the trailing edge of the ns pulse, when most of the pulse energy has already been delivered to the material. Interestingly, the reflectivity rise is preceded by a reflectivity decrease, which is caused by heating of the solid phase, since the reflectivity of the latter is a decreasing function with temperature [26]. 
The melt durations for both pulse durations can be determined by measuring the time window in which the reflectivity stays above the reflectivity of the solid phase, yielding $t=3.6 \mathrm{~ns}$ for the $\mathrm{fs}$ laser pulse and $t=6.4 \mathrm{~ns}$ for the ns laser pulse. While these values are different, they appear to be too short for considering LIPSS erasure through surface tension, smoothing ripples on a long-lived melt. This scenario was proposed by [30] who estimated threshold values of $t=200 \mathrm{~ns}$ for this process to occur. Our results suggest therefore that the absence of HSFL-\| for ns laser pulses is not a consequence of a long-lived liquid phase, underlining the above expressed hypothesis of an ultrafast, non-linear origin of HSFL-\|.

\section{Erasing fs-LIPSS upon ns laser pulse irradiation}

In view of their subtle surface morphology, small modulation depth and absence of ablation in the induced LIPSS by fs-laser exposure, the possibility of erasing them by exposure to nanosecond laser pulses was explored. The approach consisted in using a single ns pulse to locally melt a thin surface layer over a sufficiently long time to level out any ripple structures by exploiting the high surface tension of the molten phase. For this experiment, a series of spots with LIPSS were generated with fs pulses $\mathrm{F}=142 \mathrm{~mJ} / \mathrm{cm}^{2}$ and $\mathrm{N}=200$ at normal incidence, as shown in Figure 6a. These parameters were chosen to generate a variety of structures, including dual LIPSS in the outer ring and LSFL in the central disk. In order to avoid resonant enhancement of these LIPSS by the nanosecond laser pulse (also able to trigger formation of LSFL as shown in the previous section) the erasure experiment was performed at $52^{\circ}$ angle of incidence angle. The significantly different angle of incidence ensures that, if present, a periodic intensity modulation due to interference of the laser light with a surface wave will have a very different period [24] and will not enhance the existing ripples. As can be seen in Figure 6b, upon exposure to a single pulse at a high laser fluence the complete LIPSS spectrum consisting in the dual-LIPSS in the outer ring and the LSFL in the central disk could be completely erased, leaving behind a darker but homogenous surface. The laser fluence of the ns pulse was chosen to lie below the single pulse ablation threshold of Ge under that conditions. This suggests that homogeneous melting and solidification of all fs laserinduced structures has been induced, erasing the entire LIPSS landscape due to the high surface tension of the long-lived molten phase, whose lifetime we have measured with the real time reflectivity setup to be $t=83$ ns (results not shown). These results point out the possibility to perform write-read-erasure operations for data storage using fs dual-LIPSS in Ge. Multi-level, erasable storage can be envisaged by data encoding in form of four different states, namely HSFL$\|$, dual (HSFL-\|+LSFL), LSFL, and no LIPSS.

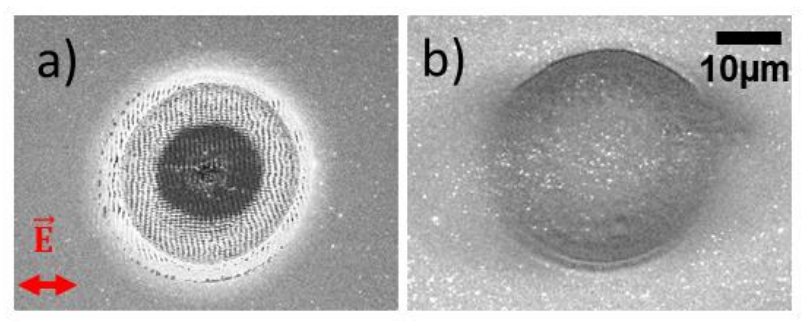

Figure 6. SEM images of fs laser irradiated regions before and after an erasure operation with a single nanosecond pulse. a) fs laser irradiation with $\mathrm{F}=142 \mathrm{~mJ} / \mathrm{cm}^{2}$ and $\mathrm{N}=200, \theta=0^{\circ}$. b) after 
exposure to a single nanosecond pulse with $F=587 \mathrm{~mJ} / \mathrm{cm}^{2}$ at $\theta=52^{\circ}$. The red arrow indicates the laser polarization direction.

\section{CONCLUSIONS}

We report on the formation of a new kind of LIPSS in Ge upon exposure to multiple pulse (N > 50) fs laser irradiation, so-called HSFL-\|, in addition to the well-known LSFL. The main characteristics of HSFL- $\|$ are a sub-wavelength ripple period of $\Lambda_{\|}=400 \pm 50 \mathrm{~nm}$, half of the laser wavelength and parallel orientation to the laser polarization direction. HSFL- $\|$ feature an extraordinary smoothness with a shallow modulation depth $d<30 \mathrm{~nm}$, which suggests that the formation process involves melting, material displacement and re-solidification, without material removal. By increasing the pulse number to $\mathrm{N}=200$, orthogonal LSFL emerge, superimposing onto the HSFL- $\|$ ripples, effectively forming dual LIPSS. The latter consist of nanohills arranged in a cubic structure, separated by the corresponding periods of the two LIPSS types. We also demonstrate the viability of suppressing HSFL-\| ripples and homogeneously extending LSFL structures in 2D by sample scanning. Interestingly, LSFL can also be generated by ns pulses, at similar fluences and number of pulses as for fs pulses. However, this is not possible for the case of HSFL-\| ripples, which are absent after ns laser exposure, which underlines the importance of ultrafast excitation to the formation of HSFL-\|. Time-resolved reflectivity measurements reveal melting of a very shallow surface layer $(<20 \mathrm{~nm})$ and short melt durations for both pulse durations. This result suggests that the absence of short-period HSFL-\| for ns laser pulses is not caused by relaxation/smoothing of high-spatial frequency surface features through a long-lived melt. Finally, we demonstrate the viability of erasing fs-laser-induced dual LIPSS by exposure to single nanosecond pulses, which points out the feasibility of performing write-read-erasure operations in dual-LIPSS in Ge for information storage applications.

\section{AUTHOR INFORMATION}

\section{Corresponding Author}

*E-mail: noemi.casquero@csic.es (N.C.).

*E-mail: j.siegel@,csic.es (J.Si.).

\section{ACKNOWLEDGMENT}

The authors are grateful to S. Sanchez-Cortes at Instituto de Estructura de la Materia of the CSIC for performing micro-Raman measurements. JSi and JSo acknowledge financial support through the national research grant UDiSON (TEC2017-82464-R) from the Spanish Research Agency (AEI, Ministry of Research and Innovation) and the European Regional Development Fund (ERDF), as well as the Consejo Superior de Investigaciones Científicas for the intramurales project (201850E057). The authors acknowledge a pre-doctoral fellowship from the MICINN for NCa and a pre-doctoral grant for RZa from the regional government of Madrid and financed by the European Social Fund (ESF) and the national "Iniciativa de Empleo Juvenil" (YEI). The authors acknowledge EU-H2020 grant agreement No 654360 (NFFA-Europe) and the corresponding access to SEM and AFM facilities at FORTH within NFFA project ID 507.

\section{REFERENCES}


[1] Bonse J, Hohm S, Kirner S V., Rosenfeld A and Kruger J 2017 Laser-Induced Periodic Surface Structures- A Scientific Evergreen IEEE J. Sel. Top. Quantum Electron. 23 9000615

[2] Gnilitskyi I, Derrien T J-Y, Levy Y, Bulgakova N M, Mocek T and Orazi L 2017 Highspeed manufacturing of highly regular femtosecond laser-induced periodic surface structures: physical origin of regularity Sci. Rep. 78485

[3] Sanz M, Rebollar E, Ganeev R A and Castillejo M 2013 Nanosecond laser-induced periodic surface structures on wide band-gap semiconductors Appl. Surf. Sci. 278 325-9

[4] Martínez-Calderon M, Azkona J J, Casquero N, Rodríguez A, Domke M, Gómez-Aranzadi M, Olaizola S M and Granados E 2018 Tailoring diamond's optical properties via direct femtosecond laser nanostructuring Sci. Rep. 814262

[5] Bonse J and Krüger J 2010 Pulse number dependence of laser-induced periodic surface structures for femtosecond laser irradiation of silicon J. Appl. Phys. 108034903

[6] Jwad T, Penchev P, Nasrollahi V and Dimov S 2018 Laser induced ripples' gratings with angular periodicity for fabrication of diffraction holograms Appl. Surf. Sci. 453 449-56

[7] Caizhen Y, Yayun Y, Baoshen J, Yuan L, Renjie D, Yong J, Yuxin W and Xiaodong Y 2017 Polarization and fluence effects in femtosecond laser induced micro/nano structures on stainless steel with antireflection property Appl. Surf. Sci. 425 1118-24

[8] Gräf S and Müller F A 2015 Polarisation-dependent generation of fs-laser induced periodic surface structures Appl. Surf. Sci. 331 150-5

[9] Kobayashi T, Wakabayashi T, Takushima Y and Yan J 2019 Formation behavior of laserinduced periodic surface structures on stainless tool steel in various media Precis. Eng. 57 $244-52$

[10] Zhang D and Sugioka K 2019 Hierarchical microstructures with high spatial frequency laser induced periodic surface structures possessing different orientations created by femtosecond laser ablation of silicon in liquids Opto-Electronic Adv. 2 19000201-18

[11] Garrelie F, Colombier J-P, Pigeon F, Tonchev S, Faure N, Bounhalli M, Reynaud S and Parriaux O 2011 Evidence of surface plasmon resonance in ultrafast laser-induced ripples Opt. Express 199035

[12] Chang C-L, Cheng C-W and Chen J-K 2019 Femtosecond laser-induced periodic surface structures of copper: Experimental and modeling comparison Appl. Surf. Sci. 469 904-10

[13] Öktem B, Pavlov I, Ilday S, Kalaycıoğlu H, Rybak A, Yavaş S, Erdoğan M and Ilday F Ö 2013 Nonlinear laser lithography for indefinitely large-area nanostructuring with femtosecond pulses Nat. Photonics 7 897-901

[14] Bonse J, Krüger J, Höhm S and Rosenfeld A 2012 Femtosecond laser-induced periodic surface structures J. Laser Appl. 2442006

[15] Le Harzic R, Dörr D, Sauer D, Neumeier M, Epple M, Zimmermann H and Stracke F 2011 Formation of periodic nanoripples on silicon and germanium induced by femtosecond laser pulses Phys. Procedia 12 29-36

[16] Reif J, Costache F, Henyk M and Pandelov S V. 2002 Ripples revisited: non-classical morphology at the bottom of femtosecond laser ablation craters in transparent dielectrics Appl. Surf. Sci. 197-198 891-5

[17] Lin Z, Liu H, Ji L, Lin W and Hong M 2020 Realization of $\sim 10 \mathrm{~nm}$ Features on Semiconductor Surfaces via Femtosecond Laser Direct Patterning in Far Field and in Ambient Air Nano Lett. 20 4947-52

[18] He S, Nivas J J, Anoop K K, Vecchione A, Hu M, Bruzzese R and Amoruso S 2015 Surface 
structures induced by ultrashort laser pulses: Formation mechanisms of ripples and grooves Appl. Surf. Sci. 353 1214-22

[19] Liu J, Jia X, Wu W, Cheng K, Feng D, Zhang S, Sun Z and Jia T 2018 Ultrafast imaging on the formation of periodic ripples on a Si surface with a prefabricated nanogroove induced by a single femtosecond laser pulse Opt. Express 266302

[20] Ehrhardt M, Lai S, Lorenz P and Zimmer K 2020 Guiding of LIPSS formation by excimer laser irradiation of pre-patterned polymer films for tailored hierarchical structures Appl. Surf. Sci. 506144785

[21] Zazo R, Solis J, Sanchez-Gil J A, Ariza R, Serna R and Siegel J 2020 Deep UV laser induced periodic surface structures on silicon formed by self-organization of nanoparticles Appl. Surf. Sci. 520146307

[22] Young J F, Preston J S, van Driel H M and Sipe J E 1983 Laser-induced periodic surface structure. II. Experiments on Ge, Si, Al, and brass Phys. Rev. B 27 1155-72

[23] Borowiec A and Haugen H K 2003 Subwavelength ripple formation on the surfaces of compound semiconductors irradiated with femtosecond laser pulses Appl. Phys. Lett. 82 4462-4

[24] Fuentes-Edfuf Y, Garcia-Lechuga M, Puerto D, Florian C, Garcia-Leis A, Sanchez-Cortes S, Solis J and Siegel J 2017 Coherent scatter-controlled phase-change grating structures in silicon using femtosecond laser pulses Sci. Rep. 7 1-12

[25] Puerto D, Garcia-Lechuga M, Hernandez-Rueda J, Garcia-Leis A, Sanchez-Cortes S, Solis J and Siegel J 2016 Femtosecond laser-controlled self-assembly of amorphous-crystalline nanogratings in silicon Nanotechnology 27265602

[26] Chaoui N, Siegel J, Solis J and Afonso C N 2001 Reflectivity of crystalline Ge and Si at the melting temperature measured in real time with subnanosecond temporal resolution $J$. Appl. Phys. 89 3763-7

[27] Ginzburg P, Fortuño F J R, Wurtz G A, Dickson W, Murphy A, Morgan F, Pollard R J, Iorsh I, Atrashchenko A, Belov P A, Kivshar Y S, Nevet A, Ankonina G, Orenstein M and Zayats A V. 2013 Manipulating polarization of light with ultrathin epsilon-near-zero metamaterials Opt. Express 2114907

[28] Siegel J, Solis J, Afonso C N and García C 1996 Bulk solidification and recalescence phenomena in amorphous Ge films upon picosecond pulsed laser irradiation J. Appl. Phys. 80 6677-82

[29] Emmony D C, Howson R P and Willis L J 1973 Laser mirror damage in germanium at 10.6 um Appl. Phys. Lett. 23 598-600

[30] Ehrlich D J, Brueck S R J J and Tsao J Y 1982 Time-resolved measurements of stimulated surface polariton wave scattering and grating formation in pulsed-laser-annealed germanium Appl. Phys. Lett. 41 630-2

[31] Fauchet P M and Siegman A E 1983 Observations of higher-order laser-induced surface ripples on 〈111〉 germanium Appl. Phys. A Solids Surfaces 32 135-40

[32] Vega F, Afonso C N and Solis J 1993 Real time optical diagnostics of the plume dynamics during laser ablation of germanium in an oxygen environment J. Appl. Phys. 73 2472-6

[33] Lin X, Li X, Zhang Y, Xie C, Liu K and Zhou Q 2018 Periodic structures on germanium induced by high repetition rate femtosecond laser Opt. Laser Technol. 101 291-7

[34] Austin D R, Kafka K R P, Lai Y H, Wang Z, Zhang K, Li H, Blaga C I, Yi A Y, DiMauro L F and Chowdhury E A 2016 High spatial frequency laser induced periodic surface structure formation in germanium by mid-IR femtosecond pulses J. Appl. Phys. 120143103 
[35] Chaoui N, Siegel J, Wiggins S M and Solis J 2005 Pressure-induced transient structural change of liquid germanium induced by high-energy picosecond laser pulses Appl. Phys. Lett. 86221901

[36] Jellison G E 1992 Optical functions of GaAs, GaP, and Ge determined by two-channel polarization modulation ellipsometry Opt. Mater. (Amst). 1 151-60

[37] Liu J M 1982 Simple technique for measurements of pulsed Gaussian-beam spot sizes Opt. Lett. 7196

[38] Florian C, Déziel J-L, Kirner S V., Siegel J and Bonse J 2020 The Role of the Laser-Induced Oxide Layer in the Formation of Laser-Induced Periodic Surface Structures Nanomaterials 10147

[39] Kafka K R P, Austin D R, Li H, Yi A Y, Cheng J and Chowdhury E A 2015 Time-resolved measurement of single pulse femtosecond laser-induced periodic surface structure formation induced by a pre-fabricated surface groove Opt. Express 2319432

[40] Fuentes-Edfuf Y, Sánchez-Gil J A, Garcia-Pardo M, Serna R, Tsibidis G D, Giannini V, Solis J and Siegel J 2019 Tuning the period of femtosecond laser induced surface structures in steel: From angled incidence to quill writing Appl. Surf. Sci. 493 948-55 